\title{
DESA: A decentralized, efficient and selective aggregation scheme in AMI
}

DOI:

10.1109/ISGT.2014.6816376

\section{Document Version}

Accepted author manuscript

Link to publication record in Manchester Research Explorer

\section{Citation for published version (APA):}

Mustafa, M. A., Zhang, N., Kalogridis, G., \& Fan, Z. (2014). DESA: A decentralized, efficient and selective aggregation scheme in AMI. In 2014 IEEE PES Innovative Smart Grid Technologies Conference, ISGT 2014/IEEE PES Innovative Smart Grid Technol. Conf., ISGT IEEE Computer Society . https://doi.org/10.1109/ISGT.2014.6816376

\section{Published in:}

2014 IEEE PES Innovative Smart Grid Technologies Conference, ISGT 2014|IEEE PES Innovative Smart Grid Technol. Conf., ISGT

\section{Citing this paper}

Please note that where the full-text provided on Manchester Research Explorer is the Author Accepted Manuscript or Proof version this may differ from the final Published version. If citing, it is advised that you check and use the publisher's definitive version.

\section{General rights}

Copyright and moral rights for the publications made accessible in the Research Explorer are retained by the authors and/or other copyright owners and it is a condition of accessing publications that users recognise and abide by the legal requirements associated with these rights.

\section{Takedown policy}

If you believe that this document breaches copyright please refer to the University of Manchester's Takedown Procedures [http://man.ac.uk/04Y6Bo] or contact uml.scholarlycommunications@manchester.ac.uk providing relevant details, so we can investigate your claim.

\section{OPEN ACCESS}




\section{DESA: A Decentralized, Efficient and Selective Aggregation Scheme in AMI}

\author{
Mustafa A. Mustafa and Ning Zhang \\ School of Computer Science \\ The University of Manchester \\ Oxford Road, Manchester, M13 9PL, UK \\ Email: \{mustafm,nzhang\}@cs.man.ac.uk
}

\author{
Georgios Kalogridis and Zhong Fan \\ Toshiba Research Europe Limited \\ Telecommunications Research Laboratory \\ 32 Queen Square, Bristol, BS1 4ND, UK \\ Email: \{george, zhong.fan $\} @$ toshiba-trel.com
}

\begin{abstract}
In this paper we propose a decentralized, efficient and selective aggregation (DESA) scheme for secure and privacypreserving communication in AMI. Unlike existing aggregation schemes, DESA uses a multi-recipient system model that is more applicable to liberalized electricity markets. It uses the homomorphic Paillier cryptosystem to encrypt users' consumption data, which are selectively aggregated by local gateways. In this way, the aggregated data of different subsets of users are only delivered to their intended authorized entities. DESA adopts the BLS signature and batch verification methods to reduce computation and communication overheads. Through security analysis and performance evaluation, we show that DESA resists various attacks and preserve users' privacy in an efficient manner.

Index Terms-smart grid, AMI, security, privacy, aggregation,

\begin{tabular}{llll}
\hline AMI & advanced metering infrastructure & SM & smart meter \\
DCC & data communication company & ACD & aggregated CD \\
DNO & distribution network operator & ECD & encrypted CD \\
TSO & transmission system operator & AECD & aggregated ECD \\
CD & electricity consumption data & GW & gateway \\
BAN & building area network & BG & BAN GW \\
NAN & neighbourhood area network & NG & NAN GW \\
WAN & wide area network & WG & WAN GW \\
\hline
\end{tabular}
\end{abstract}

\section{INTRODUCTION}

Advanced metering infrastructure (AMI) is a component of the emerging smart grid (SG) that enables remote communications of metering data [1]. In the automated meter reading, a smart meter (SM) measures its user's consumption data (CD) during a short time slot and sends it to authorised entities. However, uncontrolled access to fine-grained CD may put users' privacy at risk. Entities that access CD may use nonintrusive load monitoring techniques [2] to build individual users' consumption patterns, thus breaching their privacy.

One way to preserve users' privacy is to aggregate users' $C D$ and only make the aggregated CD (ACD) available to entities, assuming that the ACD provides sufficient information to such entities. Also nodes that aggregate the data should not access CD. This can be achieved by using homomorphic encryption techniques [3] that allow nodes to perform a specific linear algebraic operation on ciphertexts, which is equivalent to a different operation conducted on the corresponding plaintexts.

This research is supported by the Engineering and Physical Science Research Council (EPSRC) and Toshiba Research Europe Limited.
Existing aggregation schemes [4]-[8] assume that there is only a single recipient of ACD of all the users. However, in a liberalized electricity market there are multiple entities that have the legitimate right to access ACD of different sets of users. A naïve approach would be to apply one of the existing schemes multiple times, and this will amplify the overheads introduced into AMI. Therefore, there is a need for a new aggregation method more suited to a multi-recipient system model. Also, considering the large number of SMs anticipated, it is desirable to distribute the task of data aggregation across multiple entities in the grid. This distributed approach to data aggregation can also reduce communication overheads in AMI.

In this paper, we propose a novel decentralized, efficient and selective aggregation (DESA) scheme for secure communication in AMI. DESA supports selective aggregation of CD in respective users' suppliers and locations, so that only authorised entities can access the fine-grained ACD necessary for their respective dealings (enhancing users' privacy protection).

The rest of the paper is organized as follows: §II discusses the related work. §III provides the preliminaries and §IV the building blocks for the design. The DESA scheme is presented in $\S \mathrm{V}$, followed by its security analysis in $\S \mathrm{VI}$ and performance evaluation in $\S$ VII. We draw conclusions in $\S$ VIII.

\section{RELATED WORK}

There are a number of proposals on the use of a homomorphic cryptosystem for privacy-preserving data aggregation. For example, Li et al. [4] proposed an in-network aggregation scheme that uses SMs to aggregate users' encrypted CD (ECD) en route for an authorised entity, but their scheme only protects against passive attacks. Deng et al. [5] overcame this by signing each ECD. Li et al. [6] further improved [5] by using the Boneh-Lynn-Shacham (BLS) signature scheme that allows a batch verification of signatures. To reduce overheads, $\mathrm{Lu}$ et al. [7] proposed a scheme that packs user's multidimensional CD into a single ECD, whereas Ruj et al. [8] proposed a decentralized aggregation method. All of these schemes use a singlerecipient system model (inefficient for liberalized markets) and are vulnerable to passive attacks by authorised entities. If an authorised entity eavesdrops user's ECD prior aggregation, it can recover user's CD. Different from these related works, our scheme uses a multi-recipient system model. 
TABLE I. NOTATIONS

\begin{tabular}{|c|c|}
\hline $\begin{array}{c}\mathrm{d}_{j}, \mathrm{~s}_{u} \\
\mathrm{sm}_{i}, t_{n}, \mathrm{E}_{\mathrm{sm}_{i}}^{t_{n}} \\
\mathrm{E}^{t_{n}} \\
\mathrm{E}_{\mathrm{d}_{j}}^{t_{n}}, \mathrm{E}_{\mathrm{s}_{u}}^{t_{n}} \\
\mathrm{E}_{\mathrm{d}_{j}, \mathrm{~s}_{u}}^{t_{n}} \\
\mathrm{E}_{\mathrm{bg}_{i}, \mathrm{~d}_{j}, \mathrm{~s}_{u}} \\
x_{i}, y_{i} \\
p k_{i}, s k_{i} \\
k_{\mathrm{bg}_{i}} \\
C_{\mathrm{sm}_{i}}, c_{\mathrm{sm}_{i}} \\
\mathrm{ID}_{i}, \sigma_{i} \\
\mathrm{~N}_{\mathrm{sm}}, \mathrm{N}_{\mathrm{bg}}, \mathrm{N}_{\mathrm{ng}} \\
\mathrm{N}_{\mathrm{wg}}, \mathrm{N}_{\mathrm{wg}, \mathrm{d}_{i}}\end{array}$ & $\begin{array}{l}j \text { th DNO } j=\left\{1, \ldots, \mathrm{N}_{\mathrm{d}}\right\}, u \text { th supplier } u=\left\{1, \ldots, \mathrm{N}_{\mathrm{s}}\right\} \\
i \text { th user's } \mathrm{SM}, n \text {th time slot, } \in \mathrm{E}^{t_{n}}, \mathrm{CD} \text { of user } i \text { at } t_{n} \\
\text { (set of CD of all the users) in the grid at } t_{n} \\
\subset \mathrm{E}^{t_{n}},(") \text { operated by } \mathrm{d}_{j}, \subseteq \mathrm{E}^{t_{n}},(") \text { supplied by } \mathrm{s}_{u} \\
=\mathrm{E}_{\mathrm{d}_{j}}^{t_{n}} \cap \mathrm{E}_{\mathrm{s}_{u}}^{t_{n}},(") \text { operated by } \mathrm{d}_{j} \text { and supplied by } \mathrm{s}_{u} \\
\text { (") operated by } \mathrm{d}_{j} \text {, supplied by } \mathrm{s}_{u} \text { and connected to bg }{ }_{i} \\
\text { secret, public key of entity } i \text { for signing, verifying } \\
\text { homomorphic public, private key pair of entity } i \\
\text { key shared between } i \text { th BG and all its child SMs } \\
\text { ciphertext generated by sm }{ }_{i} \text { using } p k_{\mathrm{d}_{j}}, k_{\mathrm{bg}_{i}} \\
\text { identity of entity } i, \text { digital signature created by } i \\
\text { number of SMs per BG, BGs per NG, NGs per WG } \\
\text { number of WGs in the whole grid, in the } j \text { th region }\end{array}$ \\
\hline
\end{tabular}

\section{Preliminaries}

\section{A. System Model}

- Operators: There is a transmission system operator (TSO) that balances the grid and $\mathrm{N}_{\mathrm{d}}$ distribution network operators (DNOs) that maintain the grid in $\mathrm{N}_{\mathrm{d}}$ different regions.

- Suppliers: There are $\mathrm{N}_{\mathrm{s}}$ number of suppliers that serve different sets of users who are located in different regions.

- Smart meter (SM): It is an advanced metering device that measures its user's CD on per time slot, $t_{n}$, basis.

- Data communication company (DCC): It collects, aggregates and communicates users' data to authorized entities.

- Networking facility: links SMs to DCC via a hierarchical structure: building area networks (BANs), neighbourhood area networks (NANs) and wide area networks (WANs).

\section{B. Threat Model and Assumptions}

- DNOs are semi-trusted (they report correct data to TSO but may manipulate data sent to suppliers) and curious (may try to learn individual users' or other DNOs' data).

- Suppliers and DCC are honest but curious. They follow protocol specifications, but may try to get as much as possible data about individual users and other suppliers.

- External entities are not trustworthy. They may eavesdrop data in transit or even intercept and alter the data.

- SMs are tamper-proof. They are sealed and no one (including their users) could tamper with them successfully.

- Channels between operators and suppliers are secure.

\section{Design Requirements}

1) Functional requirements: For each time slot, $t_{n}$ :

(F1) each DNO, $\mathrm{d}_{j}$, should access the ACD of all the users

a) operated by $\mathrm{d}_{j}$ (located in region $j$ ), i.e. $\sum\left(\mathrm{E}_{\mathrm{d}_{j}}^{t_{n}}\right)$, so it can better manage the distribution networks in its region,

b) operated by $\mathrm{d}_{j}$ and supplied by $\mathrm{s}_{u}, \sum\left(\mathrm{E}_{\mathrm{d}_{j}, \mathrm{~s}_{u}}^{t_{n}}\right)$ for $u=$ $\left\{1, \ldots, N_{s}\right\}$, so it can split the network usage fee fairly.

(F2) each supplier, $\mathrm{s}_{u}$, should access the ACD of all its

a) users, i.e. $\sum\left(E_{s_{u}}^{t_{n}}\right)$, so it can predict their demand more accurately to avoid paying imbalance penalties,

b) users operated by $\mathrm{d}_{j}$, i.e. $\sum\left(\mathrm{E}_{\mathrm{d}_{j}, \mathrm{~s}_{u}}^{t_{n}}\right)$ for $j=\left\{1, \ldots, \mathrm{N}_{\mathrm{d}}\right\}$, so it can pay the exact network usage fee to each DNO.

(F3) TSO should access the ACD of all the users in the grid, i.e. $\sum\left(\mathrm{E}^{t_{n}}\right)$, so it can balance the grid more efficiently.

\section{2) Security requirements:}

(S1) Message authenticity: A recipient should make sure that a message is unaltered, fresh and from the claimed source.

(S2) Confidentiality of users' data: Only authorized entities (TSO, DNOs, suppliers) can access users' ACD.

(S3) User privacy preservation: Individual users' CD during each time slot, $t_{n}$, should not be revealed to any entity.

(S4) Authorisation: DNOs and suppliers can only access the ACD of the users they operate and supply, respectively.

(S5) Availability: DESA should be designed such that it is resilient to denial-of-service (DoS) attacks.

\section{BUILDING BLOCKS}

\section{A. Bilinear Pairing based Signature Schemes}

The BLS short signature [9] has a length half of the size of a DSA signature for a similar level of security. It also allows the construction of an aggregate signature [10] which can be used for a batch verification of messages sent by different users.

Let $\left(G, G_{T}, q, g, e, H\right)$ be digital signature system parameters where $G$ and $G_{T}$ are cyclic groups of the same prime order $q, g \in G$ is a generator, $e: G \times G \rightarrow G_{T}$ is a bilinear map ( $e$ is efficiently computable, $e(g, g) \neq 1$ and $e\left(g^{a}, g^{b}\right)=e(g, g)^{a b}$ for all $a, b \in Z$ [11]) and $H:\{0,1\}^{*} \rightarrow G$ is a hash function.

1) BLS short signature scheme:

- KeyGen: Select randomly $x \stackrel{\mathrm{R}}{\leftarrow} Z_{q}$ and compute $y=g^{x}$. The secret key is $x \in Z_{q}$. The public key is $y \in G$.

- SigGen: Given a message $m \in\{0,1\}^{*}$ and secret key $x$, compute the signature $\sigma=H(m)^{x}$. The signature $\sigma \in G$.

- SigVer: Given the public key $y$, message $m$, and signature $\sigma$, accept if $e(g, \sigma)=e(y, H(m))$ holds.

2) Aggregate signature:

- KeyGen, SigGen: Suppose that there are $n$ distinct users. Each user $u_{i}(i=\{1, \ldots, n\})$, generates its secret key, $x_{i}$, and public key, $y_{i}$. Then, $u_{i}$ signs its message $m_{i}$ and obtains its signature, $\sigma_{i}$, as described in IV-A1.

- SigAgg: The aggregate signature is computed by multiplying the individual signatures, i.e. $\sigma^{a g g}=\prod_{i=1}^{n} \sigma_{i}$.

- SigVer: Given the $n$ users' public keys $y_{1}, \ldots, y_{n}$, all messages $m_{1}, \ldots, m_{n}$, and the aggregate signature $\sigma^{a g g}$, compute $H\left(m_{i}\right)$. Accept if all the messages are distinct and $e\left(g, \sigma^{a g g}\right)=\prod_{i=1}^{n} e\left(y_{i}, H\left(m_{i}\right)\right)$ holds.

\section{B. Paillier Cryptosystem}

Paillier cryptosystem [3] has an additive homomorphism property. It is relatively efficient and semantically secure.

- KeyGen: Choose two large prime numbers $\left(p_{1}, q_{1}\right)$. Calculate $n=p_{1} \cdot q_{1}$ and $\lambda=\operatorname{lcm}\left(p_{1}-1, q_{1}-1\right)$. Define $L(u)=(u-1) / n$. Choose a generator $g \in Z_{n^{2}}^{*}$. Calculate $\mu=\left(L\left(g^{\lambda} \bmod n^{2}\right)\right)^{-1} \bmod n$. The public key is $p k=(n, g)$ and the private key is $s k=(\lambda, \mu)$.

- Enc: Given a message $m \in Z_{n}$, choose a random number $r \in Z_{n}^{*}$. Compute the ciphertext $C=g^{m} \cdot r^{n} \bmod n^{2}$.

- Dec: Given the ciphertext $C \in Z_{n^{2}}^{*}$, recover the message $m=L\left(C^{\lambda} \bmod n^{2}\right) \cdot \mu \bmod n$. 
1) Additive homomorphism: Multiplying the ciphertexts of messages results in a ciphertext of the sum of the messages, i.e. $C\left(m_{1}\right) \cdot C\left(m_{2}\right)=g^{\left(m_{1}+m_{2}\right)} \cdot\left(r_{1} \cdot r_{2}\right)^{n} \bmod n^{2}=C\left(m_{1}+m_{2}\right)$

2) Random factor recovery: Given a message $m$, its ciphertext $C$ and the private key $s k$, the random factor $r$ used in Enc can be recovered, i.e. $r=\left(C g^{-m} \bmod n\right)^{n^{-1} \bmod \lambda} \bmod n$.

\section{THE DESA SCHEME}

\section{A. System Initialization}

1) System Parameters Setup: A trusted authority (TA) generates parameters $\left(G, G_{T}, q, g, e\right)$, defines $H$, selects randomly $x_{\text {ta }} \stackrel{\mathrm{R}}{\leftarrow} Z_{q}$ and computes $y_{\text {ta }}=g^{x_{\text {ta }}}$. The system's master secret key is $x_{\mathrm{ta}} \in Z_{q}$ and public key is $y_{\mathrm{ta}} \in G$. TA keeps $x_{\mathrm{ta}}$ secret, but publishes the system parameters, $\left\{G, G_{T}, q, g, e, y_{\mathrm{ta}}, H\right\}$.

2) Key generation \& distribution: During license acquisition DCC generates a pair of secret/public keys, $\left\{x_{\mathrm{dcc}}, y_{\mathrm{dcc}}\right\}$, and each DNO, $\mathrm{d}_{j}$, generates a pair of homomorphic public/private keys, $\left\{p k_{\mathrm{d}_{j}}, s k_{\mathrm{d}_{j}}\right\}$. During manufacturing each SM, $\mathrm{sm}_{i}$, generates a pair of secret/public keys, $\left\{x_{\mathrm{sm}_{i}}, y_{\mathrm{sm}_{i}}\right\}$. TA generates and signs a certificate for each SM, DNO and DCC. For each gateway (GW), e.g. BAN GW (BG) $i$, DCC generates a pair of secret/public keys, $\left\{x_{\mathrm{bg}_{i}}, y_{\mathrm{bg}_{i}}\right\}$, and a certificate, and signs the latter. Finally, each $\mathrm{SM} / \mathrm{GW}$ is equipped with its certificate and secret key that is kept secret and tamper-proof.

3) $S M \& G W$ installation: During installation each SM is equipped with its local BG's and regional DNO's certificates, and each GW - with the certificates of its child SMs/GWs and parent GW. Each BG, $\mathrm{bg}_{i}$, establishes a shared secret key, $k_{\mathrm{bg}_{i}}$, with its child SMs using a standard protocol such as TLS [12].

\section{B. SM Report Generation}

$\mathbf{P}_{\mathrm{SM}}$ : At the start of a slot $t_{n+1}$, each SM, sm ${ }_{i}$, constructs a message that contains its user's ECD during $t_{n}$, and sends the message to its local BG, $\mathrm{bg}_{i}$. As shown in Fig. $1, \mathrm{sm}_{i}$ :

1. reads its users' $\mathrm{CD}$ in slot $t_{n}, \mathrm{E}_{\mathrm{sm}_{i}}^{t_{n}}$, from its register,

2. encrypts $\mathrm{E}_{\mathrm{sm}_{i}}^{t_{n}}$ with its regional DNO's public key, i.e. $C_{\mathrm{sm}_{i}}=E n c_{p k_{\mathrm{d}_{j}}}\left(\mathrm{E}_{\mathrm{sm}_{i}}^{t_{n}}\right)$, to resist passive attacks.

3. encrypts $\left\{\mathrm{ID}_{\mathrm{s}_{u}} \| C_{\mathrm{sm}_{i}}\right\}$ with $k_{\mathrm{bg}_{i}}$ to resist passive attacks by authorised entities, i.e. $c_{\mathrm{sm}_{i}}=E_{k_{\mathrm{bg}_{i}}}\left(\mathrm{ID}_{\mathrm{s}_{u}} \| C_{\mathrm{sm}_{i}}\right)$.

4. constructs $M_{\mathrm{sm}_{i}}=\left\{\mathrm{ID}_{\mathrm{sm}_{i}}\left\|\mathrm{ID}_{\mathrm{bg}_{i}}\right\| \mathrm{ID}_{\mathrm{d}_{j}}\left\|c_{\mathrm{sm}_{i}}\right\| \mathrm{TS}^{t_{n}} \|\right.$ $\left.\mathrm{TS}_{\mathrm{sm}_{i}}\right\} . \mathrm{TS}^{t_{n}}$ is a time stamp used to uniquely label $t_{n}$. $\mathrm{TS}_{\mathrm{sm}_{i}}$ is a local time stamp used to resist replay attacks.

5. signs $M_{\mathrm{sm}_{i}}, \sigma_{\mathrm{sm}_{i}}=H\left(M_{\mathrm{sm}_{i}}\right)^{x_{\mathrm{sm}_{i}}}$, to resist active attacks.

6. constructs $\operatorname{msg}_{\mathrm{sm}_{i}}=\left\{M_{\mathrm{sm}_{i}} \| \sigma_{\mathrm{sm}_{i}}\right\}$ and sends it to $\mathrm{bg}_{i}$.

\begin{tabular}{|c|c|}
\hline $\begin{array}{ll}C_{\mathrm{sm}_{i}}=E n c_{p k_{\mathrm{d}_{j}}}\left(\mathrm{E}_{\mathrm{sm}_{i}}^{t_{n}}\right), & \mathbf{P}_{\mathrm{SM}} \\
c_{\mathrm{sm}_{i}}=E_{k_{\mathrm{bg}_{i}}}\left(\mathrm{ID}_{\mathrm{s}_{u}} \| C_{\mathrm{sm}_{i}}\right), \\
M_{\mathrm{sm}_{i}}=\left\{\mathrm{ID}_{\mathrm{sm}_{i}}\left\|\mathrm{ID}_{\mathrm{bg}_{i}}\right\| \mathrm{ID}_{\mathrm{d}_{j}}\left\|c_{\mathrm{sm}_{i}}\right\| \mathrm{TS}^{t_{n}} \| \mathrm{TS}_{\mathrm{sm}_{i}}\right\}, \\
\sigma_{\mathrm{sm}_{i}}=H\left(M_{\mathrm{sm}_{i}}\right)_{\mathrm{sm}_{i}}, \\
\mathrm{msg}_{\mathrm{sm}_{i}}=\left\{M_{\mathrm{sm}_{i}} \| \sigma_{\mathrm{sm}_{i}}\right\} .\end{array}$ & $\begin{array}{ll}\mathrm{sm}_{i} & \\
\mathrm{msg}_{\mathrm{sm}_{i}} & \mathrm{bg}_{i} \\
& \end{array}$ \\
\hline
\end{tabular}

Figure 1. SM report generation: the $\mathbf{P}_{\mathrm{SM}}$ processing step.

\section{Decentralized and Selective Data Aggregation}

Users' ECD are grouped based on their intended recipients and aggregated at various networks' GWs (shown in Fig. 2).
$\mathbf{P}_{\mathbf{B G}}$ : Each BG receives messages from its child SMs, verifies and groups them based on the suppliers, aggregates ECD contained in the messages in each group, and sends the aggregated ECD (AECD) to its local NG, $\mathrm{ng}_{i}$. In detail, $\mathrm{bg}_{i}$ :

1. for each received message, $\mathrm{msg}_{\mathrm{sm}_{i}}$,

a) verifies the data contained in $\mathrm{msg}_{\mathrm{sm}_{i}}$, in terms of its: $*$ freshness $\left(V^{f r}\right)$, i.e. if $\left|\mathrm{TS}_{\mathrm{bg}_{i}}-\mathrm{TS}_{\mathrm{sm}_{i}}\right| \leq t_{\Delta}$, $*$ recipient $\left(V^{r e c}\right)$, i.e. if $\mathrm{msg}_{\mathrm{sm}_{i}}$ is addressed to $\mathrm{bg}_{i}$, * sender $\left(V^{s e n}\right)$, if $\operatorname{sm}_{i}$ is one of its child SMs,

* time slot $\left(V^{t s}\right)$, i.e. if $\mathrm{TS}^{t_{n}}$ is the expected one,

b) performs a pairing operation, i.e. $e\left(y_{\mathrm{sm}_{i}}, H\left(M_{\mathrm{sm}_{i}}\right)\right)$.

2. performs a batch verification $\left(V^{\sigma^{a g g}}\right)$ of the messages,

a) computes the aggregate signature, $\sigma^{a g g}=\prod_{i=1}^{\mathrm{N}_{\mathrm{sm}}} \sigma_{\mathrm{sm}_{i}}$

b) checks if $e\left(g, \sigma^{a g g}\right)=\prod_{i=1}^{\mathrm{N}_{\mathrm{sm}}} e\left(y_{\mathrm{sm}_{i}}, H\left(M_{\mathrm{sm}_{i}}\right)\right)$.

3. decrypts $c_{\mathrm{sm}_{i}}$ to recover $\left\{\mathrm{ID}_{\mathrm{s}_{u}} \| C_{\mathrm{sm}_{i}}\right\}$ for each $\mathrm{msg}_{\mathrm{sm}_{i}}$.

4. groups and aggregates ciphertexts destined to the same supplier, $C_{\mathrm{bg}_{i}, \mathrm{~d}_{j}, \mathrm{~s}_{u}}=\prod_{i=1}^{\left|\mathrm{E}_{\mathrm{bg}_{i}, \mathrm{~d}_{j}, \mathrm{~s}_{u} \mid}\right|} C_{\mathrm{sm}_{i}}$ for $u=1, \ldots, \mathrm{N}_{\mathrm{s}}$.

5. constructs $M_{\mathrm{bg}_{i}}=\left\{\mathrm{ID}_{\mathrm{bg}_{i}}\left\|\mathrm{ID}_{\mathrm{ng}_{i}}\right\| \mathrm{ID}_{\mathrm{d}_{j}} \|\left(\mathrm{ID}_{\mathrm{s}_{1}} \|\right.\right.$ $\left.\left.C_{\mathrm{bg}_{i}, \mathrm{~d}_{j}, \mathrm{~s}_{1}}\right)\|\ldots\|\left(\mathrm{ID}_{\mathrm{S}_{\mathrm{N}_{\mathrm{s}}}} \| C_{\mathrm{bg}_{i}, \mathrm{~d}_{j}, \mathrm{~s}_{\mathrm{N}_{\mathrm{s}}}}\right)\left\|\mathrm{TS}^{t_{n}}\right\| \mathrm{TS}_{\mathrm{bg}_{i}}\right\}$, and signs it, i.e. $\sigma_{\mathrm{bg}_{i}}=H\left(M_{\mathrm{bg}_{i}}\right)^{x_{\mathrm{bg}_{i}}}$,

6. constructs $\operatorname{msg}_{\mathrm{bg}_{i}}=\left\{M_{\mathrm{bg}_{i}} \| \sigma_{\mathrm{bg}_{i}}\right\}$ and sends it to $\mathrm{ng}_{i}$.

$\mathbf{P}_{\mathbf{N G}} / \mathbf{P}_{\mathbf{W G}}$ : Each NG/WG does the same as a BG except that it processes messages from BGs/NGs and skips step 3.

$\mathbf{P}_{\text {DCC-1 }}$ : DCC performs operations similar to those in steps 1,2 and 4 in $\mathbf{P}_{\mathbf{B G}}$ except that it processes messages from WGs and aggregates the data also in terms of DNOs.

\section{Distribution and Data Access}

DCC sends selections of AECD to their intended DNOs and suppliers. DNOs recover users' ACD and send the recovered data to TSO and their intended suppliers (shown in Fig. 3).

$\mathbf{P}_{\text {DCC-2: }}$ : For each DNO, the DCC constructs a message that contains supplier-based AECD of the users in the region managed by the DNO, signs and sends it to the DNO.

$\mathbf{P}_{\text {DCC-3 }}$ : For each supplier, the DCC constructs a message that contains region-based AECD of the supplier's customers. It then signs and sends the message to the supplier.

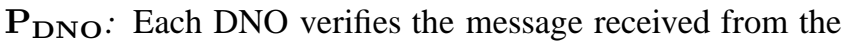
DCC, recovers the supplier-based ACD in it (satisfy (F1b)) and the random factor embedded in each AECD (as in IV-B2), and sends both items to the respective suppliers. Then, it calculates the total ACD in its region (satisfy (F1a)), and sends it to TSO.

$\mathbf{P}_{\mathbf{S}}$ : Each supplier verifies the message received from DCC and obtains the region-based AECD. It then, upon receiving a message from each DNO, recovers the ACD and random factor and computes the AECD. If the AECD computed is the same as the AECD received, it accepts the ACD (satisfy (F2b)). It then computes the ACD of all its customers (satisfy (F2a)).

$\mathbf{P}_{\text {TSO}}$ : TSO, upon receiving a message from each DNO, recovers the ACD for each DNO and then calculates the ACD of all the users in the grid (satisfy (F3)).

\section{SECURity ANALysis}

Message authenticity: Each message in DESA contains a BLS signature which is proven secure under chosen-message 


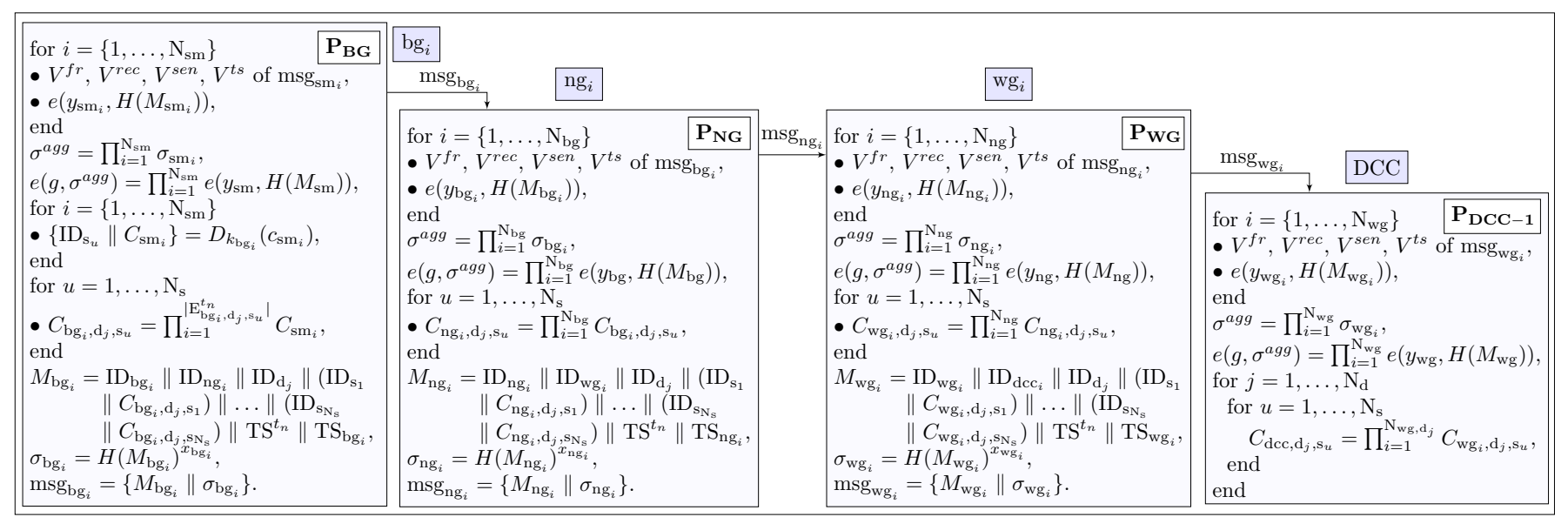

Figure 2. Decentralized and Selective Data Aggregation: the $\mathbf{P}_{\mathbf{B G}}, \mathbf{P}_{\mathbf{N G}}, \mathbf{P}_{\mathbf{W G}}$ and $\mathbf{P}_{\mathbf{D C C}-\mathbf{1}}$ processing steps.

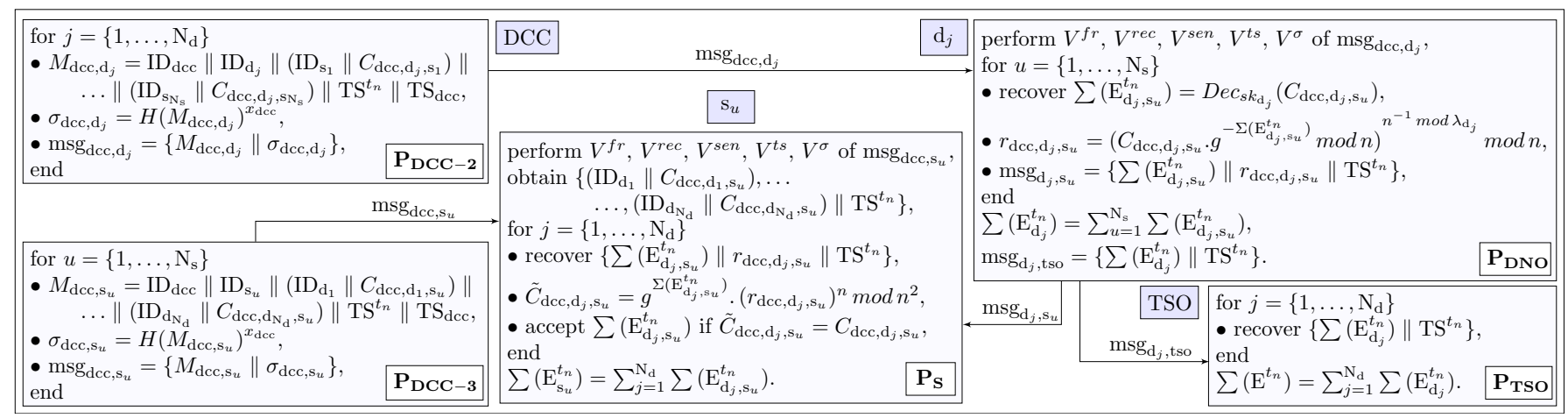

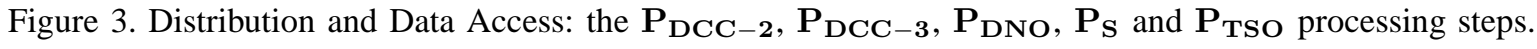

attack in the random oracle model assuming that the Computational Diffie-Hellman problem is hard [9], [10]. Hence, our scheme guaranties a source authentication, non-repudiation of origin and integrity of each message (satisfy (S1)). Any active attacks on data in transit can be detected and modified data discarded. Also, including a local time stamp in each message ensures that all such messages received are fresh.

Confidentiality: In DESA, users' CD are encrypted at SMs using the Paillier cryptosystem, then the ECD are progressively aggregated, and the AECD are delivered to DNOs, where ACD are recovered and selection of them are delivered to TSO and suppliers. As the Paillier cryptosystem is semantically secure against chosen plaintext attacks assuming that the Composite Residuosity Class problem is hard [3], only authorised entities (TSO, DNOs, suppliers) can access users' ACD (satisfy (S2)). Unauthorised internal (GWs, DCC) and external entities eavesdropping messages can only access ECD/AECD, not ACD.

User privacy-preservation: In DESA, DNOs receive only supplier-based AECD of users in their regions, thus the most fine-grained $\mathrm{CD}$ a $\mathrm{DNO} /$ supplier can access is the ACD of users located in a particular region and supplied by a particular supplier (thousands of users). Even authorized entities (TSO, DNOs, suppliers) are not allowed to access individual users' CD. DESA, unlike others, is also resistant to passive attacks by authorised entities as users' $C D$ are double encrypted (first with the regional DNO's public key, then with the key shared between the SM and its local BG) while in transit between the SM and BG. For the similar reason, if DNOs' secret keys are compromised, DESA can still operate and protect users' privacy as the most fine-grained data adversaries could decrypt is AECD sent by BGs. As long as these AECD contain ACD of sufficient number of users (several dozens), it is hard for adversaries to work out individual users' CD (satisfy (S3)).

Authorisation: In DESA, the use of selective homomorphic encryption ensures that only the entities that need to know a set of users' CD can actually access the ACD of the set, thus making DESA resistant to insider attacks (satisfy (S4)).

Availability: DESA is resistant to DoS attacks as it uses a decentralized approach to data verification/aggregation (early detection and discard of malicious messages, reducing the risk of creating a bottleneck in the system) (satisfy (S5)).

Table II presents the security level comparison of DESA.

TABLE II. SECURITY LEVEL COMPARISON

\begin{tabular}{|l|c|c|c|c|c|c|}
\hline & {$[4]$} & {$[5]$} & {$[6]$} & {$[7]$} & {$[8]$} & DESA \\
\hline (S1) Message authenticity & & $\sqrt{ }$ & $\sqrt{ }$ & $\sqrt{ }$ & & $\sqrt{ }$ \\
\hline (S2) Confidentiality & $\sqrt{ }$ & $\sqrt{ }$ & $\sqrt{ }$ & $\sqrt{ }$ & $\sqrt{ }$ & $\sqrt{ }$ \\
\hline (S3) User privacy-preservation & & & & & & $\sqrt{ }$ \\
\hline (S4) Authorisation & & & & & & $\sqrt{ }$ \\
\hline (S5) Availability & & & & & & $\sqrt{ }$ \\
\hline
\end{tabular}


TABLE III. COMPUTATION COMPLEXITY AND COMMUNICATION OVERHEAD COMPARISON

\begin{tabular}{|c|c|c|c|}
\hline & & $\overline{D E S A}$ & DSF [8] \\
\hline $\begin{array}{l}\text { computational } \\
\text { cost }\end{array}$ & $\begin{array}{l}\text { SM } \\
\text { BG } \\
\text { NG } \\
\text { WG } \\
\text { DCC }\end{array}$ & $\begin{array}{l}o_{e z}+o_{e g} \\
\left(\mathrm{~N}_{\mathrm{sm}}+1\right) * o_{p}+o_{e g} \\
\left(\mathrm{~N}_{\mathrm{bg}}+1\right) * o_{p}+o_{e g} \\
\left(\mathrm{~N}_{\mathrm{ng}}+1\right) * o_{p}+o_{e g} \\
\left(\mathrm{~N}_{\mathrm{wg}}+1\right) * o_{p}+\left(\mathrm{N}_{\mathrm{d}}+\mathrm{N}_{\mathrm{s}}\right) * o_{e g} \\
\end{array}$ & $\begin{array}{l}3 o_{e z}+3 o_{e g} \\
\left(3 \mathrm{~N}_{\mathrm{sm}}+2+\mathrm{N}_{\mathrm{s}}\right) * o_{p}+\left(2+\mathrm{N}_{\mathrm{s}}\right) * o_{e g} \\
\left(\mathrm{~N}_{\mathrm{bg}}+1\right)\left(2+\mathrm{N}_{\mathrm{s}}\right) * o_{p}+\left(2+\mathrm{N}_{\mathrm{s}}\right) * o_{e g} \\
\left(\mathrm{~N}_{\mathrm{ng}}+1\right)\left(2+\mathrm{N}_{\mathrm{s}}\right) * o_{p}+\left(2+\mathrm{N}_{\mathrm{s}}\right) * o_{e g} \\
\left(\mathrm{~N}_{\mathrm{wg}}\left(2+\mathrm{N}_{\mathrm{s}}\right)+\mathrm{N}_{\mathrm{d}}\left(1+\mathrm{N}_{\mathrm{s}}\right)+1\right) * o_{p}+\left(\mathrm{N}_{\mathrm{d}}+\mathrm{N}_{\mathrm{s}}+1\right) * o_{e g}\end{array}$ \\
\hline $\begin{array}{c}\text { communication } \\
\text { overhead }\end{array}$ & $\begin{array}{l}\text { SMs-to-BG } \\
\text { BGs-to-NG } \\
\text { NGs-to-WG } \\
\text { WGs-to-DCC }\end{array}$ & $\begin{array}{l}\mathrm{N}_{\mathrm{sm}} *(3|\mathrm{ID}|+2|\mathrm{TS}|+|\sigma|+|c|) \\
\mathrm{N}_{\mathrm{bg}} *\left(3|\mathrm{ID}|+2|\mathrm{TS}|+|\sigma|+\mathrm{N}_{\mathrm{s}} *(|\mathrm{ID}|+|C|)\right) \\
\mathrm{N}_{\mathrm{ng}} *\left(3|\mathrm{ID}|+2|\mathrm{TS}|+|\sigma|+\mathrm{N}_{\mathrm{s}} *(|\mathrm{ID}|+|C|)\right) \\
\mathrm{N}_{\mathrm{wg}} *\left(3|\mathrm{ID}|+2|\mathrm{TS}|+|\sigma|+\mathrm{N}_{\mathrm{s}} *(|\mathrm{ID}|+|C|)\right)\end{array}$ & $\begin{array}{l}3 \mathrm{~N}_{\mathrm{sm}} *(3|\mathrm{ID}|+2|\mathrm{TS}|+|\sigma|+|C|) \\
\mathrm{N}_{\mathrm{bg}} *\left(2+\mathrm{N}_{\mathrm{s}}\right) *(3|\mathrm{ID}|+2|\mathrm{TS}|+|\sigma|+|C|) \\
\mathrm{N}_{\mathrm{ng}} *\left(2+\mathrm{N}_{\mathrm{s}}\right) *(3|\mathrm{ID}|+2|\mathrm{TS}|+|\sigma|+|C|) \\
\mathrm{N}_{\mathrm{wg}} *\left(2+\mathrm{N}_{\mathrm{s}}\right) *(3|\mathrm{ID}|+2|\mathrm{TS}|+|\sigma|+|C|)\end{array}$ \\
\hline
\end{tabular}
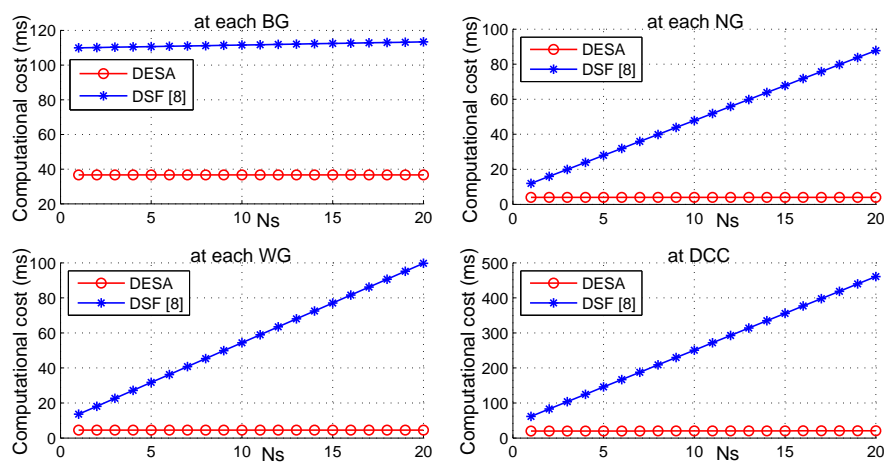

Figure 4. Computational overhead.
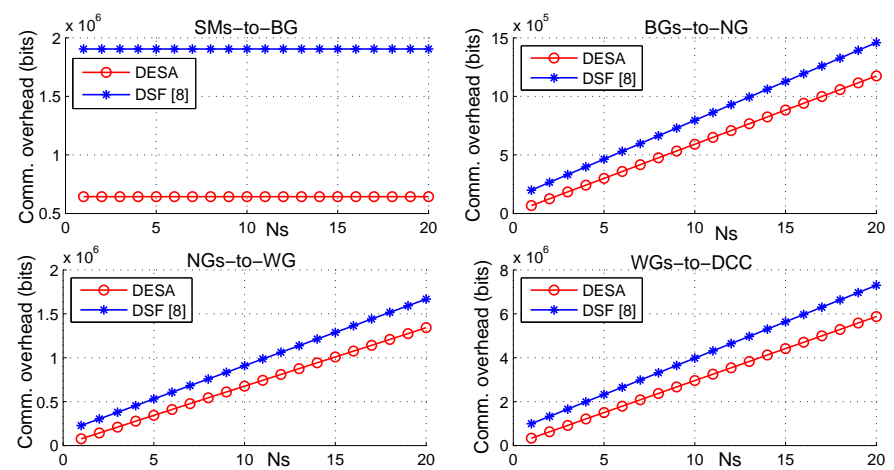

Figure 5. Communication overhead.

\section{ViI. Performance Evaluation}

In DESA, in each time slot, an SM encrypts its CD once, an $\mathrm{SM} / \mathrm{GW}$ sends one message, a GW/DCC verifies one message per child entity, and the DCC sends one message to each DNO and supplier. To meet the functional requirements, in the DSF scheme [8], an SM generates and sends ECD 3 times (to TSO, regional DNO and supplier), a GW sends $\left(2+\mathrm{N}_{\mathrm{s}}\right)$ messages (to the TSO, regional DNO and each supplier), a BG verifies 3 message per child $\mathrm{SM}$, an NG/WG/DCC verifies $\left(2+\mathrm{N}_{\mathrm{s}}\right)$ messages per child entity, and the DCC sends $\left(1+\mathrm{N}_{\mathrm{d}}+\mathrm{N}_{\mathrm{s}}\right)$ messages (to the TSO, each DNO and each supplier). Considering that computationally expensive operations are exponentiation operations in $Z_{n^{2}}\left(o_{e z}\right)$, exponentiation operations in $G\left(o_{e g}\right)$ and pairing operations $\left(o_{p}\right)$, we present the performance comparison of DESA to DSF [8] in Table III.

We ran experiments with pbc [13] and miracl [14] libraries on $3.0 \mathrm{GHz}$-processor and $4 \mathrm{~GB}$-memory machine to study the operational costs of $o_{e z}, o_{e g}$ and $o_{p}$. Our results indicate that $o_{e z}\left(\left|n^{2}\right|=2,048\right)$ costs $84.4 \mu s, o_{e g}$ in $G$ with 160 bits $43.5 \mu \mathrm{s}$, and $o_{p} 136.1 \mu \mathrm{s}$. Based on the anticipated grid size in UK, we set $\mathrm{N}_{\mathrm{sm}}=268, \mathrm{~N}_{\mathrm{bg}}=28, \mathrm{~N}_{\mathrm{ng}}=32, \mathrm{~N}_{\mathrm{wg}}=140$ and $\mathrm{N}_{\mathrm{d}}=14$. Fig. 4 depicts the variation of computational costs vs. $\mathrm{N}_{\mathrm{s}}$. Compared to [8], DESA imposes significantly less costs on each entity. We also ran simulations on Matlab with $n_{\mathrm{d}_{j}}$ 1,024-bit, $|\mathrm{ID}|(|\mathrm{TS}|)$ 32-bit and $G$ 160-bit long [7]. Fig. 5 depicts the variation of communication overheads vs. $\mathrm{N}_{\mathrm{s}}$. DESA introduces less communication overheads than [8].

\section{CONCLUSION}

We proposed a decentralized, efficient and selective aggregation scheme for use in AMI. The scheme aggregates users' $\mathrm{CD}$ in terms of users' suppliers and locations using the Paillier homomorphic cryptosystem. In contrast to existing schemes, DESA allows different authorized entities to access ACD of different subsets of users (efficiently applicable to liberalised markets). We also provided security and performance analysis of DESA to demonstrate that it provides high security strength and preserves users' privacy in efficient and scalable manner. Our future work will include an integration of electric vehicles (EVs) and EV aggregator with the SG components of DESA.

\section{REFERENCES}

[1] H. Farhangi. The path of the smart grid. Power and Energy Magazine, IEEE, 8(1):18-28, Jan.-Feb. 2010.

[2] E. L. Quinn. Privacy and the new energy infrastracture. Social Sience Research Networks (SSRN), Feb. 2009.

[3] P. Paillier. Public-key cryptosystems based on composite degree residuosity classes. In Advances in Cryptology, pages 223-238, 1999.

[4] F. Li and et. al. Secure information aggregation for smart grids using homomorphic encryption. In SmartGridComm, IEEE, Oct. 2010.

[5] P. Deng and L. Yang. A secure and privacy-preserving communication scheme for advanced metering infrastructure. In ISGT, IEEE, Jan. 2012.

[6] Fengjun Li and Bo Luo. Preserving data integrity for smart grid data aggregation. In SmartGridComm, IEEE, pages 366-371, 2012.

[7] R. Lu and et. al. Eppa: An efficient and privacy-preserving aggregation scheme for secure smart grid communications. PDS, IEEE Trans, 2012.

[8] S. Ruj and A. Nayak. A decentralized security framework for data aggregation and access control in smart grids. Smart Grid, IEEE Trans., 4(1):196-205, 2013.

[9] D. Boneh, B. Lynn, and H. Shacham. Short signatures from the weil pairing. In Advances in Cryptology, volume 2248, pages 514-532, 2001.

[10] D. Boneh and et. al. Aggregate and verifiably encrypted signatures from bilinear maps. In Advances in Cryptology, pages 416-432, 2003.

[11] D. Boneh and M. Franklin. Identity-based encryption from the weil pairing. In Advances in Cryptology, volume 2139, pages 213-229, 2001.

[12] T. Dierks and E. Rescorla. The transport layer security (tls) protocol version 1.2, August 2008. RFC 5246.

[13] Pbc library, http://crypto.stanford.edu/pbc/.

[14] Miracl library, http://certivox.org/display/ext/miracl. 